\title{
Robot-assisted surface treatment strategies of AA6014 sheet metal for accumulative roll bonding
}

\author{
Bastian Zettl and Marion Merklein
}

Bastian Zettl. Institute of Manufacturing Technology (LFT), Friedrich-Alexander-Universität Erlangen-Nürnberg, Egerlandstr. 13, 91058 Erlangen, Germany. Corresponding author. E-mail address: bastian.zettl@fau.de

Marion Merklein. Institute of Manufacturing Technology (LFT), Friedrich-Alexander-Universität Erlangen-Nürnberg, Egerlandstr. 13, 91058 Erlangen, Germany

\begin{abstract}
The application of high strength aluminum sheet metal components in automotive and aviation products effectively saves material and thus weight. Material strengthening can be realized by accumulative roll bonding (ARB), which belongs to the severe plastic deformation processes. Through repeated rolling steps a multilayered sheet metal is produced, which possesses increased strength due to its ultra-fine grained microstructure. Prior to each rolling step a surface treatment via wire brushing is mandatory for removing the oxide layer and roughening the sheet surface, which enables the bonding between the unique layers during rolling. The necessary surface treatment of the sheets is not fully understood by the current state of the art. In the past, it was not possible to achieve a defined and stable surface finish, because the brushing operation was done manually. The improvement of the process stability is essential to determine the relationship between the input parameters for brushing and the resulting bond strength of multilayered ARB sheets. For this reason, a robot-controlled surface treatment is introduced. The investigated material is the precipitationhardened aluminum AA6014 with a sheet thickness of $1 \mathrm{~mm}$. A suitable brushing kinematic under constant load is implemented and its effects on the surface properties are investigated by roughness measurements. The investigation shows, that the parameter combination leads to comparable or even higher roughness values than through manual brushing. Through 16 consecutive brushing paths a homogeneous and sufficient high surface roughness is realized, which enables material bonding in the rolling step. Thus, the research results indicate, that the robot-assisted surface treatment of ARB sheet metal is a promising method for a better automation and reproducibility of the brushing and the overall ARB process.
\end{abstract}

Keywords. Accumulative Roll Bonding, Roughness, Surface Treatment, Robot-Assisted Brushing

\section{Introduction}

Accumulative roll bonding, introduced by Sato et al. in 1998 [1], is able to provide a high degree of plastic deformation. This results in distinct grain refinement [2] and thus material strengthening according to Hall [3] and Petch [4]. The ARB process is separated in three sub-processes: surface treatment, stacking of sheets and rolling. Especially the first step of surface preparation has a high importance for the later bonding strength of the multilayered sheets. Different approaches for surface treatments were already performed prior to rolling [5]. In most cases, the surface of the sheet material is modified by an abrasive treatment, which pursues two main effects. Firstly, the thinning of the oxide layer and secondly, the roughening of the surface. The reason for this can be explained by the most common theory of bond formation during ARB, the film theory [6]. It states, that the longitudinal elongation of the sheet in the roll gap causes the thin and brittle oxide layer to break up and bare metal surfaces are exposed. The previous roughening of the sheets supports this effect, which favors a locally high shear strain on the surface [7]. At the locations of contact between bare material areas, a bond can be formed by cold welding. Wire brushing has proven itself as a target-oriented surface treatment method [5]. It is able to produce a very rough surface with little experimental effort, because it is done manually. The problem with the manual method, however, is its large dependence on the brush operator. Since the topographical condition of the surface affects the bond strength of multilayered sheets [7], varying surface conditions also affect the stability of mechanical properties. Thus, the manual brushing operator is replaced by an 
Robot-assisted surface treatment strategies of AA6014 sheet metal for accumulative roll...

industrial robot by attaching the hand brush unit to a robot arm. The precisely defined brushing process allows a more resilient correlation between surface treatment parameters, the surface properties and the resulting bond strengths of multilayered sheets.

\section{Experimental Procedure}

\subsection{Material}

The material used in this work is the precipitation-hardened aluminum AA6014 in T4 condition with a thickness of $1 \mathrm{~mm}$. The alloy composition is presented in Table 1 . The brushed sheets have a length of $450 \mathrm{~mm}$ and a width of $350 \mathrm{~mm}$.

Table 1. Chemical composition in weight percentage of Novelis Ac-170 PX.

\begin{tabular}{l|llllllllll}
\hline Element & $\mathrm{Si}$ & $\mathrm{Fe}$ & $\mathrm{Cu}$ & $\mathrm{Mn}$ & $\mathrm{Mg}$ & $\mathrm{Cr}$ & $\mathrm{Zn}$ & $\mathrm{Ti}$ & $\begin{array}{l}\text { Others each } \\
\max .\end{array}$ & Unit \\
\hline Concentration & $0.3-0.6$ & $\max .0 .35$ & $\max .0 .25$ & $0.05-0.2$ & $0.4-0.8$ & $\max .0 .2$ & $\max 0.1$ & $\max .0 .1$ & 0.15 & Wt. \% \\
\hline
\end{tabular}

\subsection{Experimental Setup: Brushing and laser cutting}

The test setup for brushing is shown in Fig. 1 and consists of the KR 200-3 industrial robot from Kuka, the PTX800 manual brushing machine from Eisenblaetter, a custom-designed enclosure with dust extraction and a customized fixture for guiding the brushing machine on the robot arm. In order to compensate for the high dead weight of the brushing unit, two compression springs are installed below the assembly plate, so that uneven surfaces and differences in the thickness of the sheet can be compensated by the possibility of moving the brushing unit in vertical direction. Before brushing, each sheet is cleaned carefully with acetone to remove any dirt and lubricant residues.

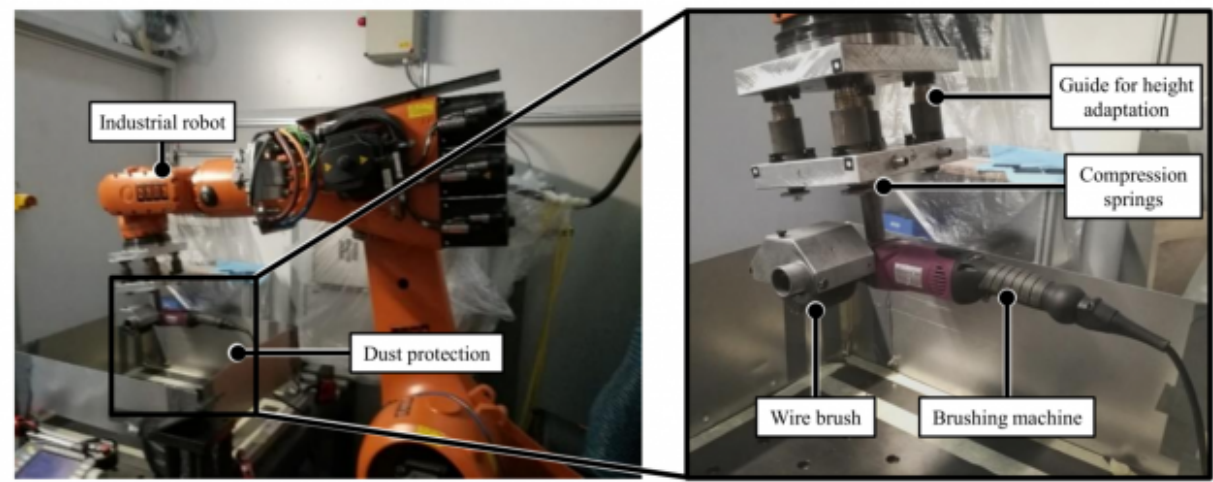

Fig. 1. Experimental setup of the robot-assisted brushing.

Fig. 2 shows the sequence of brushing paths and the relative position of the brush head to the sheet plane. By rotating the brush head by $\pm 10^{\circ}$ around the $\mathrm{z}$-axis and inverting the brushing direction by $180^{\circ}$, irregularities during contact of the brush with the sheet surface are compensated and a more homogeneous brushing result is achieved. The brushing process consists of 16 individual brushing lanes. Four adjacent paths with the $+10^{\circ}$ adjustment, followed by 
four paths with the $-10^{\circ}$ adjustment, both in rolling direction. Subsequently, again four adjacent paths with the +10 ${ }^{\circ}$ adjustment, followed by four paths with the $-10^{\circ}$ adjustment, both against rolling direction, which is implemented through rotating the brushing unit by $180^{\circ}$. For the characterization of roughness and surface topography, four identically treated sheets are processed with the following brush input parameters. The identically brushed sheets are labeled as A, B, C and D. Thus, the homogeneity of the surface properties is investigated for identical input parameters and the reproducibility of the robot-assisted surface treatment can be analyzed. For the characterization of the surface roughness, square specimens with an edge length of $50 \mathrm{~mm}$ are laser-cut out of the homogeneous brushed area of the sheets. Furthermore, metal stripes with a width of $50 \mathrm{~mm}$ and a total length of $450 \mathrm{~mm}$ are cut out of the sheets for rolling.

a) Rotation of the brush head by $\pm 10^{\circ}$ around the z-axis

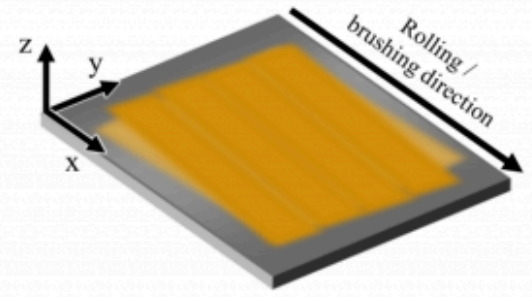

c)

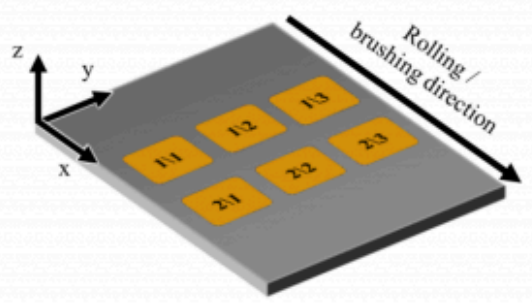

b) Inversion of brushing direction by $180^{\circ}$ and rotation of the brush head by $\pm 10^{\circ}$ around the z-axis

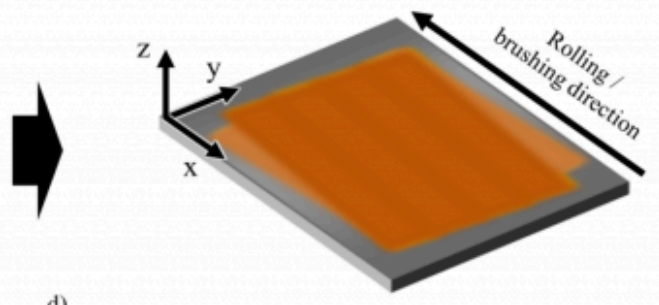

d)

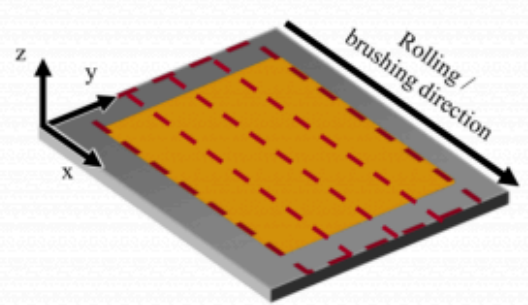

Fig. 2. Brushing steps for the robot-assisted wire brushing, with (a) showing the brushing paths 1 to 8 , (b) the brushing paths 9 to 16 and the laser cutting positions for (c) the roughness specimens and (d) the rolling stripes.

Table 2 displays the additional surface treatment parameters. A fast brushing speed of $0.14 \mathrm{~m} / \mathrm{s}$ is chosen, because it prevents over-brushing of the surface, due to the short contact dwell time of the rotating brush on the surface. In addition, the high speed and automation of the brushing process allows an overall time-saving surface treatment compared to the manual method. The length of the brush paths is determined by the longitudinal dimension of the sheet. Stainless steel is chosen as bristle material because it is harder than AA6014 and resistant to the resulting high heat input through brushing. A bristle diameter of $0.2 \mathrm{~mm}$ has proven to be suitable for surface roughening in previous investigations [5].

Table 2. Experimental parameters for the robot-assisted brushing.

\begin{tabular}{l|l|ll|ll|ll}
\hline & & \multicolumn{2}{|c|}{ Brushing roller } & \multicolumn{2}{c}{ Brushing paths } & \multicolumn{2}{c}{ Wire } \\
\hline Parameter & Brushing speed & Length & Diameter & Length & Width & Diameter & Material \\
\hline Value & $0.14 \mathrm{~m} / \mathrm{s}$ & $70 \mathrm{~mm}$ & $100 \mathrm{~mm}$ & $350 \mathrm{~mm}$ & $70 \mathrm{~mm}$ & $0.2 \mathrm{~mm}$ & V2A steel \\
\hline
\end{tabular}


Robot-assisted surface treatment strategies of AA6014 sheet metal for accumulative roll...

\subsection{Characterization of surface roughness and topography}

The characterization of roughness and surface topography is performed with both tactile and optical measurement methods. For the determination of line roughness, tactile measurements are used, since the procedure is standardized. The confocal variant is chosen because it allows larger areas of the surface to be investigated. For the tactile measurements, the Perthometer GD 120 of Mahr is used. The probe tip has a $90^{\circ}$ flank angle and a tip radius of 2 $\mu \mathrm{m}$. The vertical movement of the probe tip is recorded with inductive displacement sensors. The arithmetical mean roughness $R_{a}$, the reduced peak height $R_{p k}$, the maximum peak to valley height $R_{z}$ and the core roughness depth $R_{k}$ are calculated according to DIN EN ISO 4288 [8]. The measuring section is aligned perpendicular to the rolling direction of the brushing process. Five measurements are taken at a time, whereby the total length of the measuring section is $22.5 \mathrm{~mm}$ with a cut-off of $2.5 \mathrm{~mm}$ at the start and the end of the measured line, respectively. The surface topography is captured over a large area using the 3D confocal microscope $\mu$ surf from NanoFocus with a halogen light source. For measuring the surface roughness and capturing the topography, a 20x objective with a lateral resolution of $0.5 \mathrm{~mm}$ and a vertical resolution of $6 \mathrm{~nm}$ is used. By moving the objective with the help of the piezo drive, a large number of height sections can be recorded and a three-dimensional image of the surface is obtained. In addition, several measuring fields can be combined to cover larger areas, which allows a representative capture of the relatively coarse brush structure of the aluminum sheets. For the quantification of the surface structure, the following areal roughness parameters are taken into account: the arithmetic mean roughness $S_{a}$, the reduced peak height $S_{p k}$ and the core roughness $S_{k}$. The mean values of the areal roughness parameters are calculated by performing three large-area measurements on six samples of each identically treated sheet. This provides reliable information about the actual surface condition and the overall reproducibility of the robot-assisted brushing method.

\subsection{Rolling and characterization of bonding strength}

The rolling of the laser cut stripes is carried out at room temperature. A rolling reduction of $50 \%$ is chosen and twolayered sheets are produced by a single ARB cycle. The stripes are cut out of three robot-brushed sheets, which are roughened under the brushing parameters described above. Respectively, two stripes are stacked with the rough sides in contact and the two-layered sheets are produced. The material is prepared for peeling tests according to DIN EN ISO 11339 [9] using a tensile testing machine.

\section{Results and discussion}

\subsection{Visualization and quantification of the surface topography}

For a more descriptive illustration of the generated surface properties, Fig. 3 shows the 3D topographies acquired by confocal microscopy and the resulting false color images for sheets A and B. They are used as representatives for all tested sheets A to D, which all show equivalent results regarding the topographic structure. The topographies are recorded on six samples from the homogeneous center of every sheet. Three square measuring fields with an edge length of $2.1 \mathrm{~mm}$ are recorded for each specimen. They all show nearly identical topographies to the exemplary ones demonstrated in Fig. 3. The displayed topographies are generated by stitching together nine single images captured with the 20x objective. For quantifying the surface topography, false color images of the profile heights with square dimensions and an edge length of $1.5 \mathrm{~mm}$ are generated. A homogeneous distribution of the maxima and minima of the profile height is provided by the robot-assisted brushing. The deepest valleys of the profile are set equal zero and the maximum peaks are around $16 \mu \mathrm{m}$, which is evidence for a sufficiently high degree of surface roughening. A preferred texture direction is not recognizable, because the brushing program consists of several brushing paths in different directions and angles. 


\subsection{Characterization of the surface roughness}

Fig. 4 shows the linear roughness values $R_{a}, R_{p k}, R_{z}$ and $R_{k}$ over positions from the optically homogeneously roughened area in the center of the sheets, as displayed in Fig. 2 (c). The roughness values are determined by taking five individual line measurements per specimen, with the resulting mean value and its standard deviation. This qualifies the measured roughness parameters as a reliable source for the actual surface condition. $\mathrm{R}_{\mathrm{a}}$ varies between $2.1 \mu \mathrm{m}$ and $2.3 \mu \mathrm{m}$ over all sheets and is relatively stable over the different positions for each sheet considered individually. Meanwhile, $\mathrm{R}_{\mathrm{pk}}$ shows clearer deviations, when each sheet is considered separately. $\mathrm{R}_{\mathrm{pk}}$ varies by $\pm 15 \%$ around the average value of sheet $\mathrm{A}$ and by $\pm 10 \%$ around the average values of the remaining sheets. The mean values for sheet A, B and C are identical with $3.7 \mu \mathrm{m}$, whereas it is slightly lower for sheet D with $3.4 \mu \mathrm{m}$. Due to the stability of $\mathrm{R}_{\mathrm{a}}$ it is clear, that the height variations of the surface profiles can be reproduced with the identical surface preparation parameters. The high reduced peak height Rpk could be beneficial for ARB, because a sharp form of peaks could favor brittle cracking processes during rolling [10]. The average value of $\mathrm{R}_{\mathrm{z}}$ is $15.8 \mu \mathrm{m}$ with a deviation of $\pm 8.7 \%$ for sheet A, $16.0 \mu \mathrm{m}$ with a deviation of $\pm 4.6 \%$ for sheet B, $16.2 \mu \mathrm{m}$ with a deviation of $4.9 \%$ for sheet $\mathrm{C}$ and $15.2 \mu \mathrm{m}$ with a deviation of $5.6 \%$ for sheet D. $R_{k}$ averages between $6.2 \mu \mathrm{m}$ and $6.9 \mu \mathrm{m}$ for the sheets $A$ to $D$. $R_{z}$ is often used in ARBrelated publications as a reference for the level of surface roughening [6]. A high $\mathrm{R}_{\mathrm{Z}}$ value is beneficial for high bond strengths of the multilayered sheets. Thus, the robot-assisted surface treatment not only implements reproducibility, but also sufficiently high roughness levels. $R_{k}$ gives information about the core height of the profile, which shows stable values for the investigated sheets. In Fig. 4 (e), beside the robot-brushed sheets A to D, the mean roughness values of the hand-brushed sheets from Schödel et al. [5] are shown, with $1.7 \mu \mathrm{m}$ for $\mathrm{R}_{\mathrm{a}}$ and $10.1 \mu \mathrm{m}$ for $\mathrm{R}_{\mathrm{Z}}$, respectively. In comparison, the mean value over the robot-brushed sheets $A$ to $D$ is $22 \%$ higher for $R_{a}$ and $36 \%$ higher for $R_{z}$, which favors the robot-assisted brushing method for ARB.
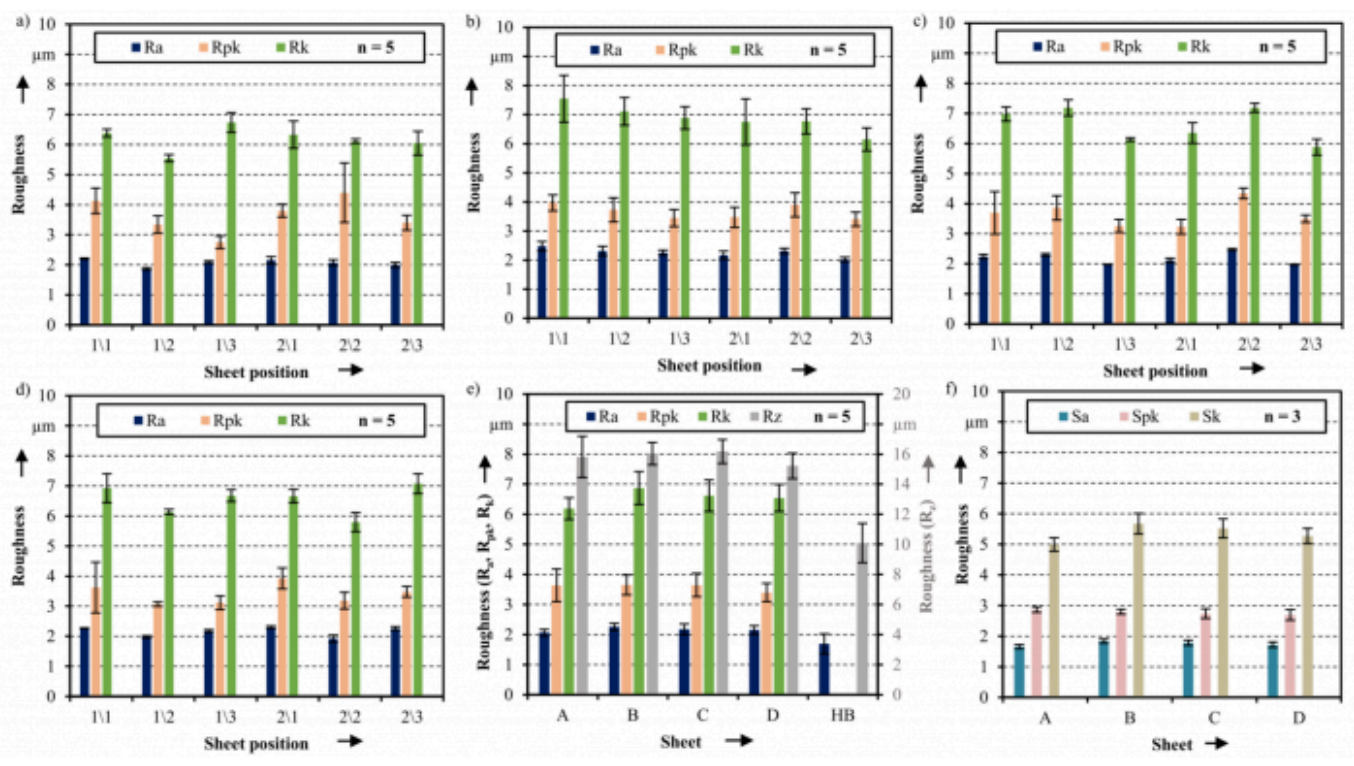

Fig. 4. Roughness parameters $R_{a}, R_{p k}, R_{k}$ and $R_{z}$ over the sheet position for (a) sheet $A$, (b) sheet $B$, (c) sheet $C$, (d) sheet D, (e) the mean values of the linear roughness parameters, including the hand-brushed sheet HB from [5] and (f) the mean values of areal roughness parameters $S_{a}, S_{p k}$ and $S_{k}$ for the sheets $A$ to $D$.

A comparison of the mean areal roughness parameters for the sheets A, B, C and D again verifies a good reproducibility of the surface treatment. The mean areal roughness values range from $1.7 \mu \mathrm{m}$ to $1.8 \mu \mathrm{m}$ for $\mathrm{S}_{\mathrm{a}}$ and from $2.7 \mu \mathrm{m}$ to $2.9 \mu \mathrm{m}$ for $\mathrm{S}_{\mathrm{pk}}$. Only $\mathrm{S}_{\mathrm{k}}$ shows slightly higher deviations over the sheet positions. The range of Sk is also larger when 
Robot-assisted surface treatment strategies of AA6014 sheet metal for accumulative roll...

comparing its mean values for the respective sheets A to D and is between values of $5.0 \mu \mathrm{m}$ and $5.7 \mu \mathrm{m}$. All areal roughness parameters are below their line roughness counterparts. However, the trend of small standard deviations and homogeneity of the surface properties for identical brushing parameters is confirmed.

\subsection{Characterization of the bonding quality}

The results of the peeling tests of the specimens PT1 to PT6 are shown in Fig. 5. A strong scattering of peeling force in the first $100 \mathrm{~mm}$ and a transition region to saturation from $100 \mathrm{~mm}$ to $200 \mathrm{~mm}$ is apparent. Thus, for the evaluation of bond strength the plateau of peel strength from $200 \mathrm{~mm}$ to $300 \mathrm{~mm}$ is used. The mean value for the peeling strength (MPS) and its standard deviation is derived from six repetitive experiments. With an average peeling strength of $0.38 \mathrm{~N} / \mathrm{mm}$ the investigated sheets are on a higher level of bond strength than hand-brushed, two-layered AA1050 aluminum sheets with an average peel strength of $0.28 \mathrm{~N} / \mathrm{mm}$ [11]. In addition, the peel strengths vary by $\pm 30 \%$, which is below the standard deviations of $\pm 35 \%$ of hand-brushed sheets in other investigations [11]. The distinct deviations can be explained by the fact, that, in case of two-layered sheets, sufficient cold welding has not taken place and the connection is more irregular due to mechanical interlocking of surface asperities [2].
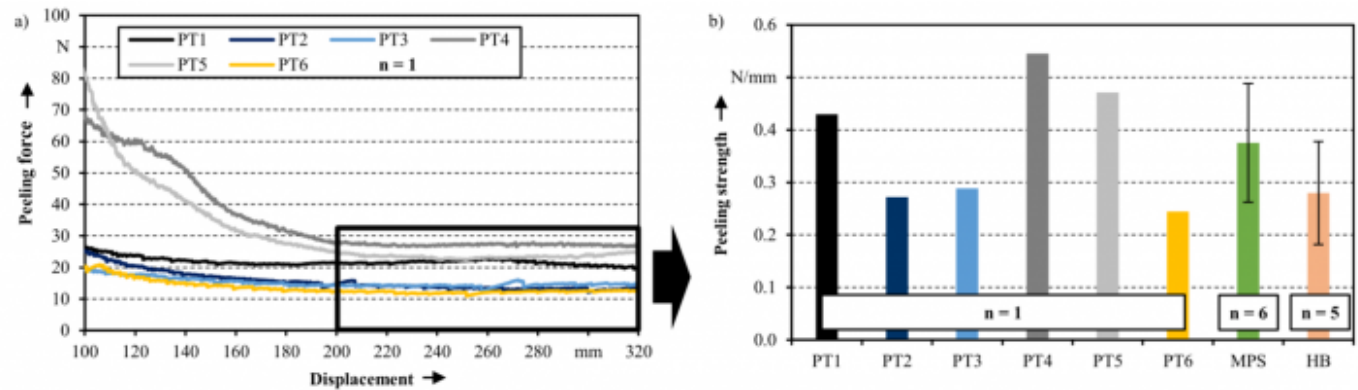

Fig. 5. Results from peeling tests with (a) peeling force as a function of displacement and (b) mean values of peeling strength in the range from $200 \mathrm{~mm}$ to $300 \mathrm{~mm}$ with the overall mean value MPS and mean peeling strength HB of hand-brushed, two-layered AA1050 sheets [11].

\section{Conclusion and outlook}

The aim of this work is to introduce the surface treatment of ARB sheets via an industrial robot as a more reproducible process compared to conventional hand brushing. After finding suitable process parameters for an optically homogeneous surface, four sheets with identical input parameters are exposed to a robot-assisted surface treatment and characterized with regard to their surface properties. The reproducibility of the surface properties could be confirmed through line and areal roughness measurements on four equally treated sheets. In a further step, it is proven that equal roughness values are resulting in identical 3D surface topographies for each sheet, which qualifies it as an additional tool for the evaluation of surface properties. The automated setting of a reproducible surface condition is a significant step for further development of the entire ARB process. In addition, the presented method of robot-brushing is more efficient in terms of process and manpower time compared to conventional hand brushing. By rolling and peeling the robot-brushed stripes, a peeling strength comparable with hand-brushed sheets but with lower deviations is identified. Thus, the robot-assisted treatment enables a systematic investigation of the correlation between surface parameters and later bond strengths of multilayered ARB products in the future. 


\section{Acknowledgements}

The authors gratefully acknowledge the German Research Foundation (DFG) for funding the research project 392174229 with the title "Improvement of the application characteristics of multi-layered sheet material for forming technology produced via accumulative roll bonding" at the Friedrich-Alexander-Universität Erlangen Nürnberg.

\section{Bibliography}

[1] Saito Y, Tsuji N. Ultra-fine grained bulk aluminum produced by accumulative roll-bonding (ARB) process. Scripta Materialia 1998;39(9):1221-7.

[2] Topić I. Ultrafine-grained metal sheets produced using the accumulative roll bonding process for light-weight structures [Doctoral Thesis]: Friedrich-Alexander- Universität Erlangen-Nürnberg (FAU); 2008.

[3] Hall EO. The Deformation and Ageing of Mild Steel: III Discussion of Results. Proceedings of the Physical Society 1951;64(9):747-53. [4] Petch N. The Cleavage Strength of Polycrystals. The Journal of the Iron and Steel Institute 1953(174):25-8.

[5] Schödel S, Merklein M. Investigation of different surface treatment parameters in the context of roll bonding processes. Procedia Manufacturing 2019;29:600-7.

[6] Tsuji N, Saito Y. ARB (Accumulative Roll-Bonding) and other new Techniques to Produce Bulk Ultrafine Grained Materials. Advanced Engineering Materials 2003;5(5):338-44.

[7] Jamaati R, Toroghinejad MR. The Role of Surface Preparation Parameters on Cold Roll Bonding of Aluminum Strips. Journal of Materials Engineering and Performance 2011;20(2):191-7.

[8] DIN EN ISO 4288:1998-04, Geometrical Product Specifications (GPS) - Surface texture: Profile method - Rules and procedures for the assessment of surface texture; German version. Berlin: Beuth Verlag GmbH.

[9] DIN EN ISO 11339:2010-06, Adhesives - T-peel test for flexible-to-flexible bonded assemblies; German version. Berlin: Beuth Verlag GmbH.

[10] Vaidyanath LR, Nicholas MG. Pressure welding by rolling. British Welding Journal 1959;6:13-28.

[11] Böhm W. Verbesserung des Umformverhaltens von mehrlagigen Aluminiumblechwerkstoffen mit ultrafeinkörnigem Gefüge: FAU University Press; 2019.

PDF automatically generated on 2021-05-20 20:08:00

Article url: https://popups.uliege.be/esaform21/index.php?id=3831

published by ULiège Library in Open Access under the terms and conditions of the CC-BY License (https://creativecommons.org/licenses/by/4.0) 